\title{
Moving Vortices Interacting with Periodic Pinning
}

\author{
Gilson Carneiro \\ Instituto de Física \\ Universidade Federal do Rio de Janeiro \\ C.P. 68528, 21945-970, Rio de Janeiro-RJ, Brasil
}

Received on 28 February, 2002

\begin{abstract}
Recent results obtained by the author for the dynamical phase diagrams for vortices in clean films, driven by an uniform force, and interacting with periodic pinning resulting from a columnar defect lattice are discussed. Using numerical simulations of a simple model and other considerations, the dynamical phase diagrams are obtained as a function of the driving force magnitude and direction, the temperature, and the vortex density. The following dynamical phases and dynamical phase transitions are found. Moving vortex lattices at low temperatures, with spatial order that can be commensurate or incommensurate with the periodic pinning, moving vortex liquids and moving smectics. Dynamical melting of moving vortex lattices into moving vortex liquids takes place and transverse pinning of moving commensurate vortex lattices and smectics occurs. It is found that the dynamical phase diagrams in the theoretical limit of infinite driving force magnitudes play a central role in determining the whole dynamical phase diagram: each dynamical phase originates from an infinite-drive limit phase with the same spatial symmetry that evolves continuously into finite-drive regions of the dynamical phase diagram. It is argued that this conclusion also applies for a large class of periodic pinning potentials.
\end{abstract}

\section{Introduction}

A problem of current interest is the study of moving vortices interacting with arrays of pinning centers. Random arrays have received the most attention. However, periodic pinning arrays are also of interest. One reason is that they provide examples of dynamical phases and phase transitions, a subject not yet fully understood, where theoretical predictions can be tested in superconducting films with artificial defect lattices[1], and in Josephson junction arrays (JJA)[2]. Earlier works on this subject are as follows.

Vortex dynamics is simpler when the driving force magnitude is large, in which case the velocity of the center of mass (CM) of the vortex array is also large, because the moving vortices average the pinning potential. In the case of moving vortices interacting with random pinning arrays the large driving force limit was first considered by Schmid and Hauger[3]. They suggested that fast moving vortices average out the pinning potential and, consequently, order in a triangular lattice at low temperatures. In experiments [4] and numerical simulations[5] nearly triangular lattices are indeed observed at large CM velocity. Koshlev and Vinokur [6] analyzed the effects of random pinning at large CM velocities on vortex displacement from the lattice equilibrium positions. They argued that it is equivalent to a "shaking" temperature, inversely proportional to the $\mathrm{CM}$ velocity, that adds to the thermodynamic tem- perature, and predicted that dynamical melting of the moving vortex lattice takes place when the CM velocity is such that the combined temperature equals the equilibrium melting temperature. Recently, Giamarchi and Le Doussal [7] pointed out that this picture for the large CM velocities behavior breaks down, because the vortices order in a moving glass, rather than in a moving triangular lattice. The reason is that averaging of the pinning potential by the fast moving vortices is only partial. A static random pinning potential remains acting on the vortices in the frame of reference moving with the $\mathrm{CM}$ velocity (CM frame), and has non-trivial consequences on vortex order. These authors predict that the vortices move along static channels, on average parallel to the direction of drive, and are pinned on them with respect to a transverse force at zero temperature. This is referred to as transverse pinning. These predictions are in agreement with experimental and numerical results [4].

In the case of vortices interacting with periodic pinning, it is also expected that at large CM velocities average of the pinning potential takes place. However, its consequences for the dynamical phase diagram have only been explored recently $[8,9]$. Previous theoretical studies on the subject are mostly numerical. Extensive studies of the zero-temperature vortex dynamics 
in systems with columnar-defect(CD) lattices, carried out by several workers [5], find a rather complex behavior, with many dynamical phases and phase transitions. A simpler finite-temperature behavior is found in JJA and films. Marconi and Domimguez [10] carried out numerical simulations of square JJA, with the driving force along the $[1,0]$ (or $[0,1]$ ) direction. They find three dynamical phases: two moving vortex lattices and a moving liquid. In all three, the CM velocity is along the direction of drive. The lattices have the same spatial order, but differ from each other by the response to a force transverse to the direction of motion. One lattice, named transversely pinned vortex lattice, is pinned (transverse pinning), and the other, named floating solid, is unpinned. Dynamical melting of the floating solid into the liquid, and dynamical depinning from the pinned lattice to the floating solid are reported. Further work [11] predicts transverse pinning only for driving forces along $[1,0]$ ( or $[0,1]$ ), and verify it experimentally. Finite-temperature work on films was carried out by Reichhardt and Zimányi[12] for vortices interacting with a square CD-lattice driven along the $[1,0]$, or $[0,1]$, directions, and for the vortex density for which there is one vortex per CD. These authors find three dynamical phases: a moving lattice, commensurate with the pinning potential periodicity along the direction transverse to motion, a moving smectic and a moving liquid. Dynamical transitions between the commensurate lattice and the smectic and between the smectic and the liquid are found, driven either by the temperature or by vortex motion.

The remaining of this paper describes the approach proposed by the author $[8,9]$ to construct the finitetemperature dynamical phase diagram for vortices interacting with a CD-lattice as a function of the magnitude and direction of the driving force, the temperature, and the vortex density.

This approach starts by pointing out that in the limit of very large $\mathrm{CM}$ velocities the moving vortices average the periodic pinning potential only in the direction of motion, and that the dynamical phases reduce to the equilibrium ones for vortices interacting with the averaged pinning potential $[8,9]$. Then it considers the limit of very large CM velocities, referred to here as the infinite-drive limit, for a generic model for vortices in clean films interacting with periodic pinning (Sec. II). It is shown that in this limit the dynamical phases reduce, in the $\mathrm{CM}$ frame, to the equilibrium ones for vortices interacting with the periodic pinning potential averaged in the direction of motion. These phases, referred to here as infinite-drive phases, stablish the exact asymptotic behavior of the dynamical phases at large values of the driving force magnitude, for a given direction of motion and temperature. The infinite-drive phases depend also on the details of the pinning potential and on the vortex density. The next step is to consider a simple model that keeps the most important features of the problem ( Sec. III). This model describes vortices in films interacting with square lattice of columnar defects (Fig. 1). First the infinite-drive limit for the model is considered. The pinning potential (Fig. 2.a) averaged in the direction of motion is nontrivial only for the $[0,1]$ (or $[1,0]$ ) and $[-1,1]$ (or $[1,-1]$ ), where it is a washboard periodic in the perpendicular direction (Fig. 2.b and c). In other directions it is either a constant or a very shallow washboard (Fig. 2.d). The washboards for $[0,1]$ (or $[1,0]$ ) and $[-1,1]$ (or $[1,-$ 1]) stabilize moving vortex lattices at low temperature that can be commensurate or incommensurate with the washboard's periodicity (Fig. 3). The infinite-drive phase diagrams are then obtained as a function of the direction of motion and temperature for two typical vortex densities by equilibrium Monte Carlo simulations. Next, numerical simulations of Langevin's stochastic dynamical equations for the model are carried out in order to determine the dynamical phase diagram at finite drives (Sec. IV). The most important result obtained from these simulations is that, as far as the spatial order is concerned, the infinite-drive phases exhaust the whole dynamical phase diagram. In other words, all dynamical phases originate from infinite-drive phases with the same spatial symmetry that evolve continuously into regions of finite drives. It is argued that, with a few exceptions, this is also true for a large class of periodic pinning potentials (Sec. V).

\section{Infinite-drive limit}

Here the infinite-drive limit is discussed for a generic model for vortices interacting with a CD-lattice. By considering Langevin's stochastic dynamical equations for this model in the limit where the driving force magnitude approaches infinity, it is shown that these equations, written in the CM frame, describe vortices interacting between themselves and with a static effective pinning potential, equal to the average of the periodic pinning potential in the direction of motion. As a consequence, the dynamical phases reduce to the corresponding thermal equilibrium ones, the infinite-drive phases, that can be distinguished from each other only by the spatial symmetry.

The motion of $N_{v}$ two-dimensional vortices is assumed to be governed by Langevin equations for massless particles, which for the $l$-th vortex reads[13],

$$
\eta \frac{d \mathbf{r}_{l}}{d t}=\mathbf{F}_{l}^{v-v}+\mathbf{F}_{l}^{v-c d l}+\mathbf{F}_{d}+\boldsymbol{\Gamma}_{l},
$$

where $\eta$ is the friction coefficient,

$$
\mathbf{F}_{l}^{v-v}=-\sum_{j \neq l=1}^{N_{v}} \nabla_{l} U^{v-v}\left(\mathbf{r}_{l}-\mathbf{r}_{j}\right),
$$

is the force of interaction with other vortices, $U^{v-v}(\mathbf{r})$ being the vortex-vortex interaction potential in twodimensions,

$$
\mathbf{F}_{l}^{v-c d l}=-\nabla_{l} U^{v-c d l}\left(\mathbf{r}_{l}\right)
$$


is the force of interaction with the CD-lattice, $U^{v-c d l}(\mathbf{r})$, being the respective potential, given by

$$
U^{v-c d l}(\mathbf{r})=\sum_{\mathbf{R}} U^{v-c d}(\mathbf{r}-\mathbf{R})
$$

where $\mathbf{R}$ denotes the CD-lattice positions and $U^{v-c d}(\mathbf{r})$ is the interaction potential between a vortex and a single $\mathrm{CD}, \mathbf{F}_{d}$ is the driving force, and $\boldsymbol{\Gamma}_{l}$ is the random force appropriate for temperature $T$. In terms of Fourier transforms $U_{l}^{v-c d l}$ can be written as

$$
U^{v-c d l}(\mathbf{r})=\sum_{\mathbf{Q}} U^{v-c d}(\mathbf{Q}) e^{i \mathbf{Q} \cdot \mathbf{r}}
$$

where $\mathbf{Q}$ denotes the CD-lattice reciprocal lattice vectors, and $U^{v-c d}(\mathbf{Q})$ is the Fourier transform of $U^{v-c d}(\mathbf{r})$.

It is convenient to consider vortex motion in the frame moving with the CM. Let $\mathbf{r}_{l}^{\prime}(t)=\mathbf{r}_{l}(t)-\mathbf{V}_{c m} \mathrm{t}$ denote the $l$-th vortex position in the CM frame, $\mathbf{V}_{c m}$ being the CM velocity, to be defined shortly. In the $\mathrm{CM}$ frame the vortex-CD lattice interaction, denoted here by $\mathbf{F}_{l}^{v-c d l}(t)$, depends explicitly on time, namely

$$
\mathbf{F}_{l}^{v-c d l}(t)=\sum_{\mathbf{Q}}(-i \mathbf{Q}) U^{v-c d}(\mathbf{Q}) e^{i \mathbf{Q} \cdot \mathbf{r}_{l}^{\prime}} e^{i \mathbf{Q} \cdot \mathbf{V}_{c m} t}
$$

The equation of motion for the $l$-th vortex in the CM frame reads,

$$
\eta \frac{d \mathbf{r}_{l}^{\prime}}{d t}=\mathbf{F}_{l}^{v-v}+\mathbf{F}_{l}^{v-c d l}(t)+\mathbf{F}_{d}-\eta \mathbf{V}_{c m}+\boldsymbol{\Gamma}_{l}
$$

The CM velocity is defined by the condition $\left.\sum_{1}^{N_{v}}\left\langle d \mathbf{r}_{l}^{\prime}(t) / d t\right)\right\rangle_{t}=0$, where \langle\rangle$_{t}$ denotes average over the random force distribution and over time. It follows from Eq. (7) that

$$
\eta \mathbf{V}_{c m}=\mathbf{F}_{d}+\frac{1}{N_{v}} \sum_{j=1}^{N_{v}}\left\langle\mathbf{F}_{l}^{v-c d l}(t)\right\rangle_{t} .
$$

In the limit $F_{d} \rightarrow \infty$, it follows from Eqs. (6) and (8) that $\mathbf{V}_{c m}=\mathbf{F}_{d} / \eta$, so that $V_{c m} \rightarrow \infty$ also. In this case, all Fourier components in $\mathbf{F}_{l}^{v-c d l}(t)$, Eq. (6), for which $\mathbf{Q} \cdot \mathbf{V}_{c m} \neq 0$ oscillate fast, having a negligible effect on the vortex trajectory [3], and $\mathbf{F}_{l}^{v-c d l}(t)$ reduces to the static force obtained by summing the Fourier components in $\mathbf{F}_{l}^{(v-c d l)}(t)$ with $\mathbf{Q} \cdot \mathbf{V}_{c m}=0$, or $\mathbf{Q} \perp \mathbf{F}_{d}$, namely

$$
\mathbf{F}_{l}^{v-c d l}(t) \rightarrow \sum_{\mathbf{Q} \perp \mathbf{F}_{\mathbf{d}}}(-i \mathbf{Q}) U^{v-c d}(\mathbf{Q}) e^{i \mathbf{Q} \cdot \mathbf{r}_{l}^{\prime}}
$$

This force derives from the static effective pinning potential

$$
U_{\alpha}^{e f f}\left(r_{\perp}^{\prime}\right)=\sum_{\mathbf{Q} \perp \mathbf{F}_{\mathbf{d}}} U^{(v-c d)}(\mathbf{Q}) e^{i Q r_{\perp}^{\prime}}
$$

where $r_{\perp}^{\prime}$ is the $\mathrm{CM}$ frame coordinate perpendicular to the direction of $\mathbf{F}_{d}$, and $\alpha$ the orientation of $\mathbf{F}_{d}$. This potential is equal to the average of $U^{v-c d l}$ in the direction of motion (and drive). By definition, $U_{\alpha}^{e f f}\left(r_{\perp}^{\prime}\right)$ is one-dimensional and periodic in the direction perpendicular to $\mathbf{F}_{d}$, if $\mathbf{F}_{d}$ is oriented along one of the square CD-lattice directions and a constant otherwise, since no $\mathbf{Q}$ is perpendicular to $\mathbf{F}_{d}$. The equations of motion in the CM frame, Eqs. (7), reduce then to those describing vortices interacting between themselves and with $U_{\alpha}^{e f f}\left(r_{\perp}^{\prime}\right)$ at temperature $T$. Their long-time solutions are the corresponding equilibrium phases, called infinite-drive phases in this paper.

The infinite-drive limit establishes the exact asymptotic behavior $F_{d} \rightarrow \infty$ of the dynamical phase for given $\mathbf{F}_{d}$ orientation and $T$. For finite $F_{d}$, the dynamical phase is expected to be close to the infinite-drive one with the same spatial symmetry for sufficiently large $F_{d}$. The condition for this to happen is that the vortex displacements in the $\mathrm{CM}$ frame caused by the pinning force oscillations in time, Eq. (7), during a time interval of the order of one-half period, are small compared with the vortex mean separation $a_{v}$. This means that for every $\mathbf{Q}$, such that $\mathbf{Q} \cdot \mathbf{F}_{d} \neq 0$,

$$
Q\left|U^{(v-c d)}(\mathbf{Q})\right| \frac{\pi}{\mathbf{Q} \cdot \mathbf{F}_{d}} \ll a_{v} .
$$

\section{Model and numerics}

Here a simple model that allows explicit results for the the dynamical phase diagrams to be obtained is considered [8]. The model considers vortices and CD placed on a square lattice (space lattice), subjected to periodic boundary conditions with $256 \times 256$ square primitive unit cells of dimensions $d \times d$. The CD-lattice is square, oriented parallel to space lattice and commensurate with it. The CD-lattice, the coordinate system and the angles defining the orientations of $\mathbf{F}_{d}(\alpha)$ and $\mathbf{V}_{c m}(\theta)$ are shown in Fig. 1 .

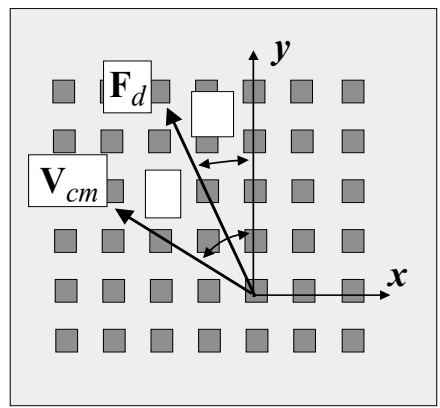

Figure 1. Columnar defect-lattice and definitions of coordinate system, CM velocity $\left(\mathbf{V}_{c m}\right)$ and driving force $\left(\mathbf{F}_{d}\right)$ orientations.

vortex has a core of linear dimension $d_{v}=4 d\left(d_{v} \sim\right.$ $2 \xi(0))$, so that each vortex occupies 16 space-lattice sites. The vortex-vortex interaction potential is a 
screened Coulomb one [14], periodic in the space lattice, and characterized by the energy scale $J=$ $\left(\phi_{0}^{2} d_{v} / 32 \pi^{3} \lambda^{2}\right)$, where $\lambda$ is the penetration depth. That is, $U^{v-v}(\mathbf{r})$ is the lattice Fourier transform of

$$
U^{v-v}(\mathbf{k})=4 \pi^{2} J \frac{e^{-\frac{\kappa^{2}}{\kappa_{c}^{2}}}}{\kappa^{2}+\Lambda^{-2}},
$$

where $\kappa^{2}=4 \sin ^{2}\left(k_{x} d / 2\right)+4 \sin ^{2}\left(k_{y} d / 2\right), k_{x}$ and $k_{y}$ being the space lattice reciprocal lattice vectors components, $\kappa_{c}=2 \sin \left(\pi d / 2 d_{v}\right)$ is the vortex core cutoff in k-space, and $\Lambda$ is the screening length $(\Lambda>\lambda)$. The square CD-lattice has $N_{c d}=8 \times 8$ sites, lattice constant $a_{c d}=32 d$. The interaction potential between a vortex and a single $\mathrm{CD}$ is chosen with depth $U^{v-c d}(\mathbf{r}=0)=-J$, range $R_{c d}=12 d$ and a spatial dependence that gives square equipotentials and a pinning force of constant modulus $F_{p}=J / R_{c d}$, as shown in Fig. 2.a. The reciprocal CD-lattice vectors are

$$
\mathbf{Q}=Q_{1}\left(n_{1} \hat{\mathbf{x}}+n_{2} \hat{\mathbf{y}}\right),
$$

where $Q_{1}=2 \pi / a_{c d}$, and $n_{1}, n_{2}=-4,-3, \ldots, 3$. It is found that the lattice Fourier transforms $U^{v-c d}(\mathbf{Q})$ are: $U^{v-c d}(0)=-0.19 J, U^{v-c d}\left( \pm Q_{1} \hat{\mathbf{x}}\right)=U^{v-c d}\left( \pm Q_{1} \hat{\mathbf{y}}\right)=$ $0.10 J, U^{v-c d}\left( \pm Q_{1}[\hat{\mathbf{x}} \pm \hat{\mathbf{y}}]\right)=-0.06$, and essentially negligible otherwise.

The model has the square lattice symmetry, so that the dynamical phase diagrams need only to be studied for $\mathbf{F}_{d}$ orientations $0^{\circ} \leq \alpha \leq 45^{\circ}$.

The dynamical phases spatial order is obtained by calculating the time-averaged density-density correlation function, $P(\mathbf{r})$, which is proportional to the probability that a vortex is found at $\mathbf{r}$, given that there is one at $\mathbf{r}=0$, and its Fourier transform, the structure function, $S(\mathbf{k})$. The motion of vortices is characterized by calculating the CM velocity, and the time-averaged velocity of each vortex.

Two types of numerical studies of the model are carried out. Equilibrium Monte Carlo simulations to obtain the infinite-drive phases, described in detail in Sec. IV A. Numerical integration of Langevin's equations to obtain the dynamical phase diagram as a function of $F_{d}, \alpha$ and $T$ for two typical vortex densities: two vortices per $\mathrm{CD}$ and five vortices per four CD. These corresponds to the magnetic inductions $B=2 B_{\phi}$ and $B=1.25 B_{\phi}$, respectively, where $B_{\phi}=\phi_{0} / a_{c d}^{2}$ is the matching field, for which there is one vortex per CD. The technical details of these simulations are given in Refs. [8, 9].

\section{Results}

In this section the simulation results for the dynamical phase diagrams are reported. First in the infinite-drive limit, then for finite drives. In the figures presented here driving force magnitudes are measured relative to the single CD pinning force $F_{p}$, Sec. III; temperatures relative to the infinite-drive moving incommensurate lattice melting temperature $T_{m}$, Sec. IV A and center of mass velocity components relative to the components of $\mathbf{V}_{d} \equiv \mathbf{F}_{d} / \eta$.

\section{A. Infinite-Drive Limit}

The infinite-drive phase diagrams are obtained by equilibrium Monte Carlo simulations of the lattice London model with the pinning potential $U_{\alpha}^{e f f}\left(r_{\perp}\right)$. It is found that for $\mathbf{F}_{d}$ along $[0,1]\left(\alpha=0^{\circ}\right)$ and [-1,1] $\left(\alpha=45^{\circ}\right)$ the $U_{\alpha}^{e f f}\left(r_{\perp}\right)$ are the washboards shown in Figs. 2b) and c). For $\mathbf{F}_{d}$ along other lattice directions, the $U_{\alpha}^{e f f}\left(r_{\perp}\right)$ are found to be very shallow washboards, because the $U^{(v-c d)}(\mathbf{Q})$ (Eq. (10)) are very small (Sec. III), and are considered as constant potentials in this paper. For example, the $U_{\alpha}^{e f f}\left(r_{\perp}\right)$ for $\mathbf{F}_{d}$ along the [$1,2]$ direction $\left(\alpha=26.6^{\circ}\right)$, shown in Fig. 2.d has a well depth more than one order of magnitude smaller than that for $\alpha=0^{\circ}$ and $45^{\circ}$ shown in Figs. 2b) and c). For $\mathbf{F}_{d}$ oriented in non-lattice directions, $U_{\alpha}^{e f f}\left(r_{\perp}\right)$ is a constant.
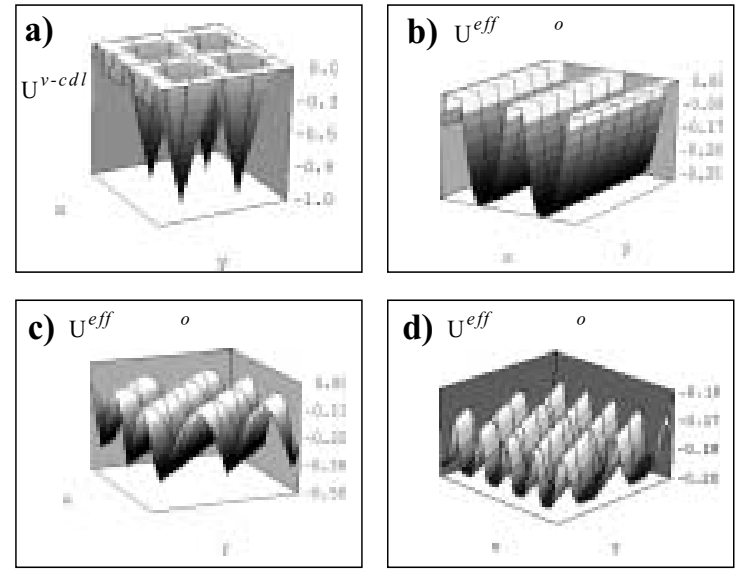

Figure 2. a) Columnar defect-lattice pinning potential. b-d) Effective pinning potentials for motion in: b) $[0,1]$ direction $\left(\alpha=0^{\circ}\right)$, c) $[-1,1]$ direction $\left(\alpha=45^{\circ}\right)$, and d) [-1,2] direction $\left(\alpha=26.6^{\circ}\right)$. Potentials in units of the single CD potential well depth $(J)$.

The corresponding low- $T$ infinite-drive phases are as follows. For $\alpha=0^{\circ}$ and $45^{\circ}$, they are vortex lattices (VL) commensurate or incommensurate with the one-dimensional periodicity, depending on $B$. The VL for $\alpha=0^{\circ}$, are obtained here as a function of $B$, for $B \geq B_{\phi}$. Their density-density correlation functions, $P(\mathbf{r})$, are shown in Fig. 4 . The commensurate lattices consist of identical vortex chains within each washboard channel, with neighbor chains displaced with respect to each other by half a chain period. There is a single chain for $B_{\phi} \leq B \leq B_{1}$, and two chains for $B_{1}<B \leq B_{2}$. The values of $B_{1}$ and $B_{2}$ are found to be in the ranges 
$B_{1}<1.125 B_{\phi}$ and $1.125 B_{\phi}<B_{2}<1.375 B_{\phi}$. A commensurate-incommensurate transition takes place for $B=B_{c-i}\left(1.25 B_{\phi}<B_{c-i}<1.375 B_{\phi}\right)$. For $B>B_{c-i}$ the VL is incommensurate, and nearly triangular. For $B=2 B_{\phi}$ and $B=1.25 B_{\phi}$ the $P(\mathbf{r})$ shown in Fig. 4 correspond, respectively, to the vortex lattices labeled MIL and MCL0 in Fig. 3. The effect of the one-dimensional periodic potential on the incommensurate lattice is to displace the vortices from the triangular lattice positions by a distance small compared to the lattice parameter[15]. It is found that, as expected, this effect is greater for $B$ in the vicinity of $B_{c-i}$. This is evidenced by the behavior of $P(\mathbf{r})$ shown in Fig. 4. For $B=1.375 B_{\phi}, 1.5 B_{\phi}$, and $1.75 B_{\phi}, P(\mathbf{r})$ has smeared spots centered in a nearly triangular grid, with smearing increasing with distance. For $B>2 B_{\phi}$, $P(\mathbf{r})$ has sharp spots, indicating that the displacements are negligible, and the VL is sharply defined.
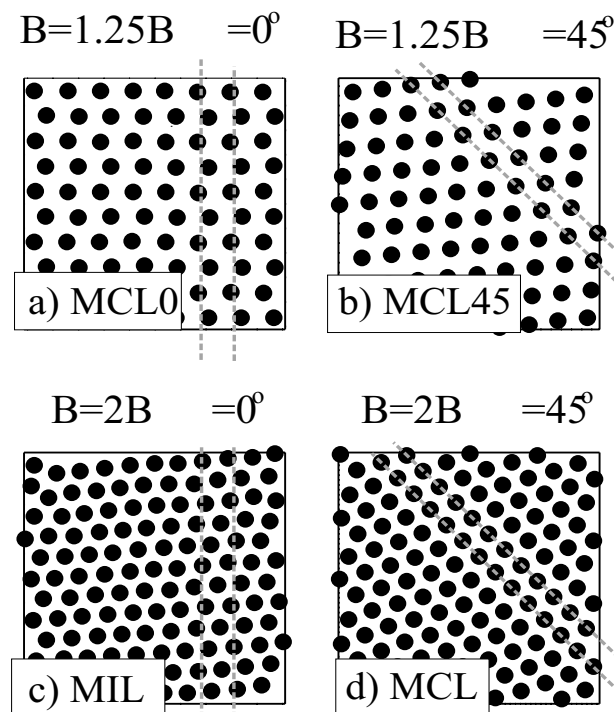

Figure 3. Vortex positions in low- $T$ infinite-drive phases. Dashed gray lines indicate minima of $U_{\alpha}^{e f f}$ shown in Figs.2.b and 2.c. Nomenclature: moving commensurate lattices: a) MCL0, b) MCL45 and d) MCL. Moving incommensurate lattices: c) MIL

The two $B$ values studied in this paper, $B=2 B_{\phi}$ and $B=1.25 B_{\phi}$, are typical of parameter regions far from commensurate-incommensurate transitions, both for $\alpha=0^{\circ}$ and $\alpha=45^{\circ}$, where the VL are sharply defined. The infinite-drive phase diagrams in these cases are as follows. For $\alpha=0^{\circ}$ and $45^{\circ}$ the VL are shown in Fig. 3. These are referred to in this paper as moving incommensurate lattices (MIL) and moving commensurate lattices, with distinct vortex configurations labeled MCL, MCL0 and MCL45, as shown in Fig. 3. For $0^{\circ}<\alpha<45^{\circ}$, the VL are incommensurate and nearly triangular. It is found that the incommensurate lattices for the same $B$, but different $\alpha$, cannot be dis- tinguished from each other. Hereafter all incommensurate lattices are referred to as moving incommensurate lattices (MIL).

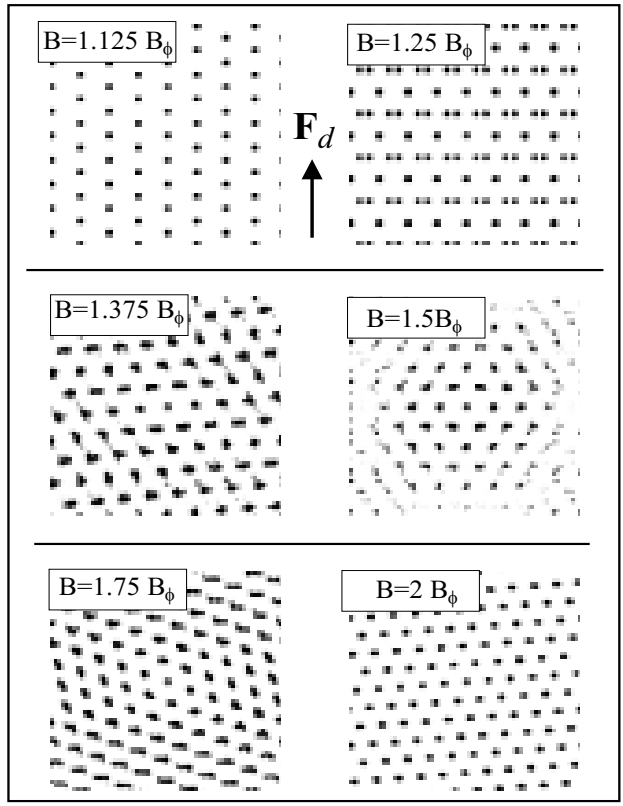

Figure 4. Density-density correlation functions, $P(\mathbf{r})(\mathbf{r}=0$ is at the panel's center), for low- $T$ infinite-drive phases for motion in the $[0,1]\left(\alpha=0^{\circ}\right)$ direction as a function of $B$.

The $T$-dependence of the infinite-drive phases (at constant $\alpha$ is found to be as follows: the moving incommensurate lattices (MIL) melts into a moving vortex liquid (MLQ) at $k_{B} T_{m}=0.09 \mathrm{~J}$, for both $B$. For $B=$ $2 B_{\phi}$ the moving commensurate lattice (MCL) changes into a moving smectic (MSM) at $T_{m c l} / T_{m}=1.4$ and the moving smectic (MSM) changes into a moving liquid (MLQ) at $T_{m s m} / T_{m}=1.8$. For $B=1.25 B_{\phi}$ the moving commensurate lattices, MCL0 and MCL45, change into a moving smectic (MSM) at $T_{m c l 0} / T_{m}=1.2$ and $T_{m c l 45} / T_{m}=1.7$, respectively.

\section{Drive along $\alpha=0^{\circ}$ and $\alpha=45^{\circ}$}

Dynamical phase diagrams $\left(F_{d}\right.$ vs. $\left.T\right)$ are shown in Fig. 5. For $B=2 B_{\phi}, \alpha=0^{\circ}$, only two dynamical phases exist: a moving incommensurate lattice (MIL) and a moving liquid (MLQ), separated by a dynamical melting line, as shown in Fig. 5. The dynamical phase diagrams for both $B, \alpha=45^{\circ}$, and for $B=1.25 B_{\phi}, \alpha=$ $0^{\circ}$, are similar to one another, containing three phases: a moving commensurate lattice (MCL for $B=2 B_{\phi}$, MCL0 and MCL45 for $B=1.25 B_{\phi}$ ) a moving smectic (MSM) and a moving liquid. Only the dynamical phase diagrams for $B=2 B_{\phi}, \alpha=45^{\circ}$, and $B=1.25 B_{\phi}$, $\alpha=0^{\circ}$ are shown in Fig. 5. That for $B=1.25 B_{\phi}$, $\alpha=45^{\circ}$, is similar. In the temperature range covered by Fig. 4 the transitions between the moving smectics and moving liquids do not appear. In Fig. 5 typical 
$P(\mathbf{r})$ at large $F_{d} / F_{p}$ for the above described phases are shown. It is found that the dynamical phases at large $F_{d} / F_{p}$ essentially coincide with the corresponding infinite-drive ones. The dynamical phase diagrams in Fig. 5 show clearly that each dynamical phase originates from the infinite-drive one with the same spatial symmetry that evolves continuously into finite drive regions.

As discussed next, the commensurate VL (MCL, MCL0 and MCL45) show transverse pinning at low $T$. This may be expected because for $\alpha=0^{\circ}$ and $\alpha=45^{\circ}, \mathbf{V}_{c m}$ remains parallel to $\mathbf{F}_{d}$. In all these cases, according to Eq. (6), the effective pinning potential $\left(U_{\alpha}^{e f f}\left(r_{\perp}\right)\right)$ acts on the moving vortices for all $F_{d}$, not only in the $F_{d} \rightarrow \infty$ limit. The commensurate phases are pinned by $U_{\alpha}^{e f f}\left(r_{\perp}\right)$ with respect to a small force along the direction defined by $r_{\perp}$.
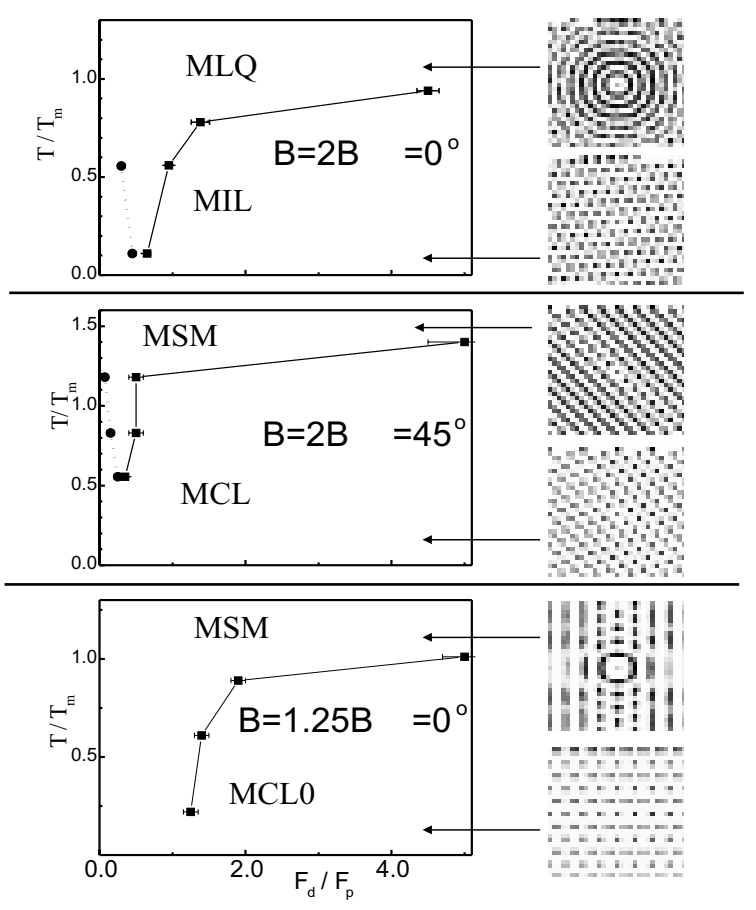

Figure 5. Dynamical phase diagrams for drives along $[0,1]$ $\left(\alpha=0^{\circ}\right)$ and $[-1,1]\left(\alpha=45^{\circ}\right)$. Panels: density-density correlation functions $P(\mathbf{r})$ at high drives $(\mathbf{r}=0$ is at the panel's center). Nomenclature: $\mathrm{MLQ}=$ moving liquid, $\mathrm{MSM}=$ moving smectic. Others as in Fig.3. Dotted lines in the pannels for $B=2 B_{\phi}$ indicate where vortex motion stops.

2. Drives along $0^{\circ}<\alpha<45^{\circ}$ The dynamical phases

found in this range of driving-force orientations have the same spatial symmetries as those described in Secs. IV A and IV A 1. and are referred to in what follows by the same nomenclature.
The $F_{d}$ vs. $\alpha$ dynamical phase diagrams at low- $T$ are shown in Fig. 6. Both have two dynamical transition lines. One, referred to here as dynamical melting line, separating a moving incommensurate lattice (MIL) and a moving liquid (MLQ). Another separating a moving commensurate lattice (MCL for $B=2 B_{\phi}$ and MCL45 for $B=1.25 B_{\phi}$ ) or a moving smectic (MSM) for $B=2 B_{\phi}$ and a moving liquid. Within the moving commensurate lattice and moving smectic regions transverse pinning occurs, with the vortices moving along the $[-1,1]$ direction. The transition line in the $B=2 B_{\phi}$ dynamical phase diagram from the moving commensurate lattice (MCL) and the moving smectic (MSM) occurs with the vortices moving in the $[-1,1]$ direction, and is essentially identical to that for $\alpha=45^{\circ}$ discussed in Sec. IV A 1.

The evidence leading to the construction of the dynamical phase diagrams in Fig. 6 is discussed in detail in Refs.[8, 9]
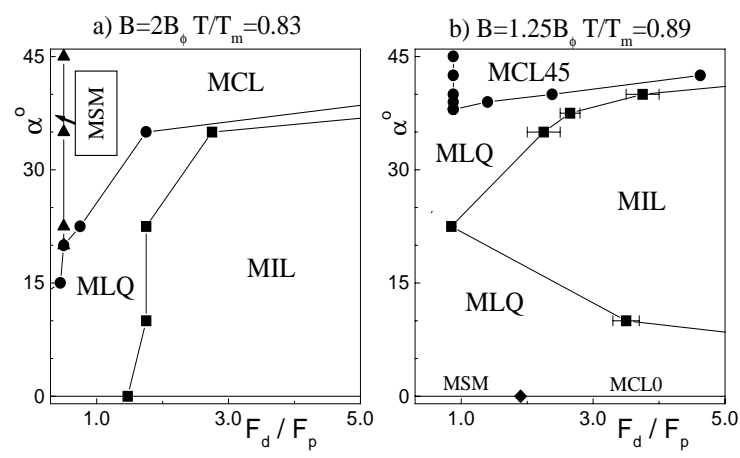

Figure 6. Dynamical phase diagrams for $T=$ constant. Dynamical phases named as in Figs. 3 and 4.

The detailed properties of the dynamical phase diagrams shown in Fig. 6 are as follows.

i) DynamicalMelting. The dynamical melting lines extend from $\alpha=0^{\circ}$ to $\alpha=45^{\circ}$. For $B=2 B_{\phi}$ it touches the $\alpha=0^{\circ}$-axis at the $F_{d}$ value where the dynamical melting takes place for $\alpha=0^{\circ}$ and $T / T_{m}=0.83$ (Fig. $5)$. It does not touch the $\alpha=45^{\circ}$ axis for both $B$ and the $\alpha=0^{\circ}$ axis for $B=1.25 B_{\phi}$, because the dynamical phases in these axes are not moving incommensurate lattices, as discussed in Sec. IV A. These result of (Fig. 6) show that, for both $B$, the moving incommensurate and commensurate lattices are separated by a moving liquid, at least up to the highest $F_{d}$ studied here. However, in the infinite-drive phase diagram there is no moving liquid at these temperatures. It is unclear how the disappearance of the moving liquid as $F_{d} \rightarrow \infty$ takes place.

The $\theta$ vs. $F_{d}$ curves in Figs. 7 and 8 show that in the moving incommensurate lattice (MIL) the directions of drive and vortex motion are approximately equal $(\theta \simeq \alpha)$, and that the melting transition is reflected in this curve by a change in slope. For $B=2 B_{\phi}$ 
this change is sharp, with $\theta$ increasing rapidly towards $\theta=45^{\circ}$ as $F_{d}$ decreases. For $B=1.25 B_{\phi}$ the same is true, as long as $\alpha \gtrsim 20^{\circ}$, while for smaller $\alpha$ the moving liquid $\theta$ slowly approaches $\theta=0^{\circ}$ as $F_{d}$ decreases.

ii) TransversePinning. The results shown in Figs. 7 and 8 indicate that in the regions where the spatial order is that of a moving commensurate lattice, or a moving smectic for $B=2 B_{\phi}$, transverse pinning occurs, and vortex motion is restricted to the $[-1,1] \mathrm{di}-$ rection. This is seen in Figs. 7 and 8 , where $V_{\perp}=0$ and $\theta=45^{\circ}$ in the $F_{d}$ range of moving commensurate lattices or smectics, and the distribution of individual vortices direction of motion is sharply peaked around $\theta=45^{\circ}$ (Fig. 7, inset). These results also show that the moving commensurate and smectic regions evolve continuously from the corresponding infinite-drive phases.

The temperature dependence of the dynamical phase diagrams is as follows. For temperatures lower than those of Fig. 6 the dynamical phase diagrams are similar. The dynamical melting lines are shifted to lower $F_{d}$, similarly to what happens for $B=2 B_{\phi}$ and $\alpha=0^{\circ}$ (Fig. 5). Transverse pinning in the moving commensurate lattices also occurs, as evidenced by the $V_{\perp}$ vs. $F_{d}$ curves shown in Fig. 9 . For higher temperatures several changes are observed. The dynamical melting lines also exist, shifted to larger $F_{d}$, provided $T$ does not exceed the infinite-drive melting temperature $T_{m}$. When $T>T_{m}$ the moving incommensurate lattices do not exist, only moving liquids.
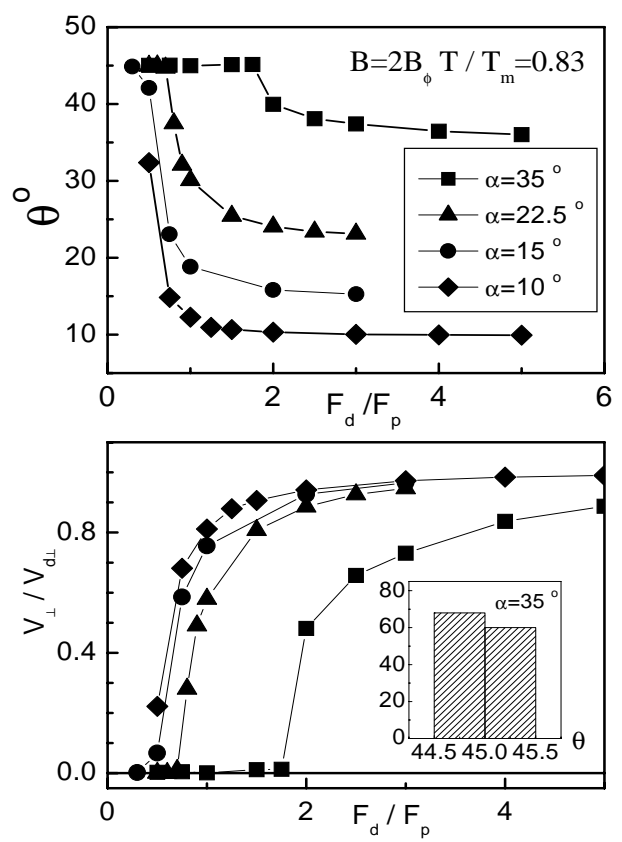

Figure 7. Center of mass velocity direction $(\theta)$ and component perpendicular to the $[-1,1]$ direction , $V_{\perp}$, vs. driving force magnitude along lines of constant $\alpha$ in Fig.5.a. Inset: histogram for the distribution of vortices direction of motion $(\theta)$ for $\alpha=35^{\circ}$ and $F_{d} / F_{p}=1.5$.

As well known[7], transverse pinning in the moving commensurate lattices or smectics only exists, strictly speaking, at $T=0$. At finite $T$ thermal excitation of lattice defects lead to flux motion away from the $[-1,1]$ direction, giving rise to a finite $V_{\perp}$. The regions of transverse pinning found in the present simulations occur because the thermally excited $V_{\perp}$ is smaller than the simulation resolution. In experiments, where the resolution is also finite, these regions may also be present. The $T$ dependence of the $V_{\perp}$ vs $F_{d}$ curves are shown in Fig. 9. For $B=2 B_{\phi}$ transverse pinning is found in the moving commensurate lattice and moving smectics at temperatures above $T_{m}$, whereas for $B=1.25 B_{\phi}$ it disappears at lower temperatures.
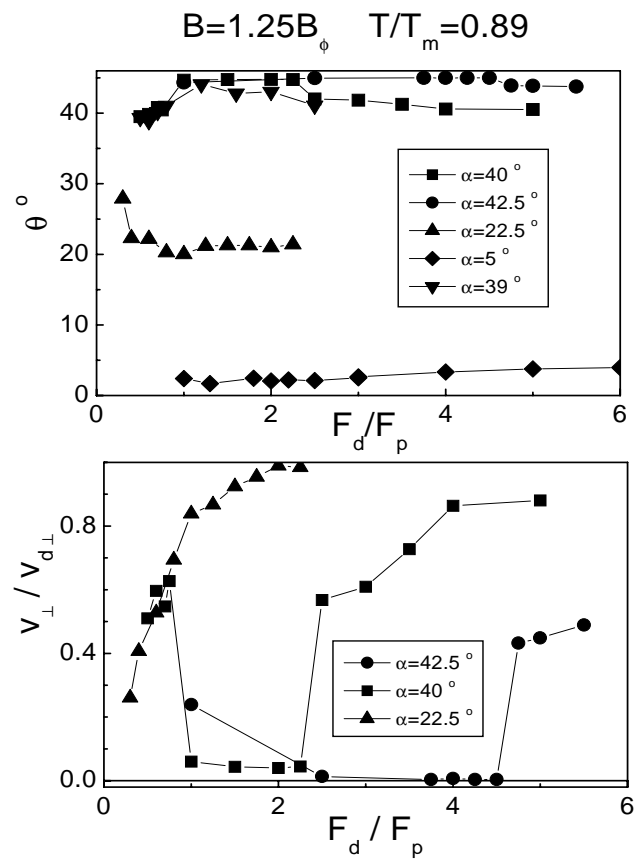

Figure 8. Center of mass velocity direction $(\theta)$ and component perpendicular to the $[-1,1]$ direction,$V_{\perp}$, vs. driving force magnitude along lines of constant $\alpha$ in Fig.5.b.

\section{Discussion}

The results described above reveal the important role played by the infinite-drive phases. They show that all dynamical phases originate from infinite-drive phases with the same spatial symmetry that evolve continuously into regions of finite drives.

The results obtained by Reichhardt and Zimányi[12] for vortices in films interacting with a square CDlattice, driven in the $[1,0]$ direction, at $B=B_{\phi}$, show a similar behavior. The infinite-drive phase diagram was not considered in this paper, but can be identified with the reported dynamical phase diagrams at high drives. It consists, in the terminology adopted here, of the following phases. A moving commensurate lattice at low 
$T$, with single-chain structure similar to that shown in Fig. 4 for $B=1.125 B_{\phi}$ rotated by $90^{\circ}$, a moving smectic at intermediate $T$, and a moving liquid at high $T$. The dynamical phase diagram reported in Ref.[12] shows that these three phases exist in continuous regions of the driving force vs. $T$ phase diagrams that extend from high to low drives. This phase diagram is similar to those found here for $B=1.25 B_{\phi}, \alpha=0^{\circ}$ and $45^{\circ}$, and for $B=2 B_{\phi} \alpha=45^{\circ}$ (Sec. IV A 1).
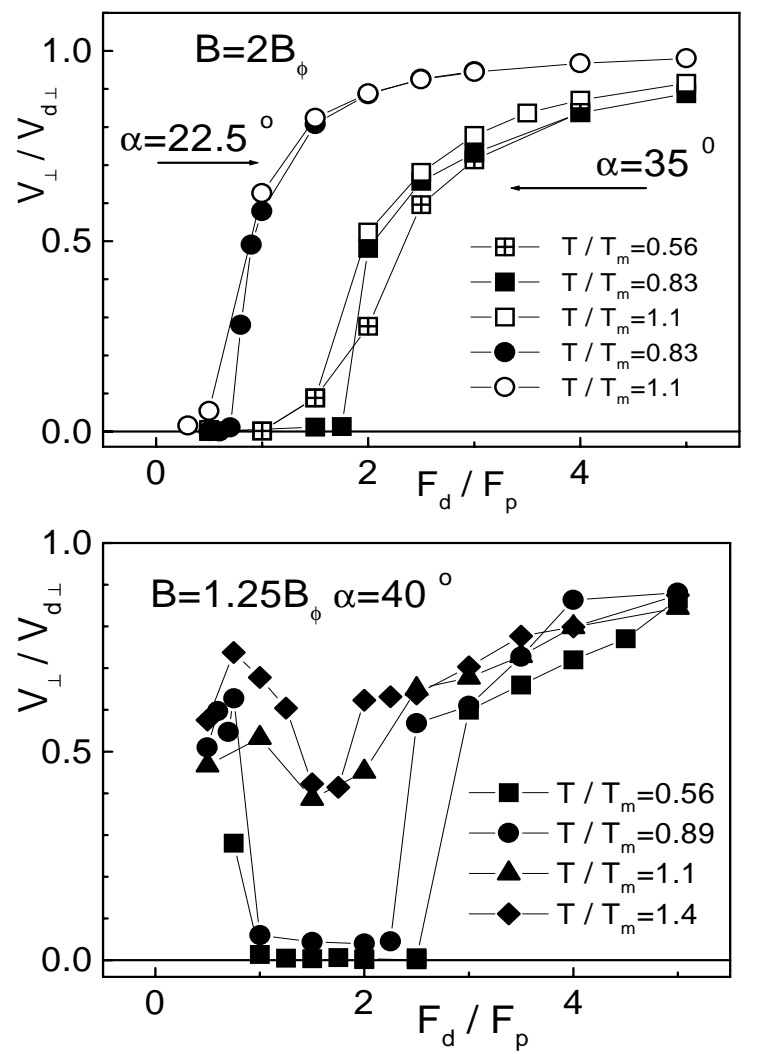

Figure 9. Component of $\mathrm{CM}$ velocity perpendicular to [$1,1], V_{\perp}$, vs. driving force magnitude along lines of constant $\alpha$ and at constant temperatures.

For vortices in JJA, driven in the $[1,0]$ direction, the dynamical phase diagram reported by Marconi and Dominguez[10] also show dynamical phases existing in continuous regions of the driving force vs. $T$ plane that extend from high to low drives. However it is not possible to identify the infinite-drive phases from the data reported in the paper.

Some studies of vortices interacting with CDlattices at $T=0$ [5] find dynamical phases and phase transitions similar to the ones describes here and in Ref.[12]. Examples are tranverse pinning, moving commensurate and incommensurate lattices and dynamical transitions between them. However, these studies probe dynamical behavior different from the finitetemperature ones discussed here. At finite temperatures, the vortices relax after sufficient long times to a steady state, identified as the dynamical phase, that is unique as far as the probability distribution is concerned [16]. Accordingly, dynamical phase changes caused by varying the driving force, the temperature, or both, are reversible. This is not necessarily the case at $T=0$. However, in numerical studies, irreversibility appears due to insufficient run times to reach the steady state, particularly when relaxation times are very large. This occurs in several circumstances, such as low temperatures, or vicinity of dynamical phase changes. In the simulations reported in this paper low temperatures are avoided. Some simulation runs where performed cycling the driving force magnitude from a large value to a small one and back. Small hysteresis is found near dynamical phase transitions.

In conclusion, the approach descried here to construct the dynamical phase diagrams, starting from the infinite-drive ones, provides a simple method, based on equilibrium statistical mechanics, to identify dynamical phases spatial order, and to predict dynamical phase transitions. It is expected that this method is applicable to a large class of periodic pinning potentials and vortex densities. The reasons are that the effective pinning potentials resulting from averaging physically reasonable periodic pinning potentials in the direction of motion are, as those discussed in Sec. IV A, essentially constant for most directions, and one-dimensional and periodic for a few particular ones. This predicts the existence, for $B \geq B_{\phi}$, of moving lattices, commensurate or incommensurate with the effective pinning potential periodicity, moving smectics and moving liquids, similar to the ones reported here, and of dynamical phase transitions between then. For $B<B_{\phi}$ the moving lattices may be different, particularly at low vortex densities, where the commensurate infinite-drive phases are not expected to retain the simple chain structure found here for the $B \geq B_{\phi}$ ones. However, the close relationship between the dynamical phase diagrams and the infinite-drive ones are still valid, as evidenced by the JJA results of Ref.[10]. The dynamical phases obtained by this method do not, in general, exhaust the dynamical phase diagram. One known type of dynamical phase that has no corresponding infinite-drive phase is that in which some vortices are pinned by the periodic potential and others are moving. These are found at low drives in some $T=0$ simulations for $B>B_{\phi}$ [5]. No such phases are found in the present simulations nor in Refs.[5, 10]. The details of how the dynamical phases and dynamical phase transitions predicted by the method proposed here fit into the dynamical phase diagram for each particular model and vortex density depends in a complicated way on the model parameters, and has to be determined in each case.

\section{Acknowledgments}

Research supported in part by CNPq, CAPES, FAPERJ, and FUJB. 


\section{References}

[1] A.N. Lykov, Adv. Phys. 42, 263 (1993) V.V. Metlushko et al., Sol. St. Comm. 91, 331 (1994); M. Baert et al., Phys. Rev. Lett. 74, 3269 (1995); E. Rossel et al., Phys. Rev. B 53, R2983 (1996); V.V. Moshchalkov et al., ibid. 54, 7385 (1996); 57, 3615 (1998); K. Harada et al., Science 274, 1167 (1996). J.-Y. Lin et al., Phys. Rev. B 54, R12717 (1996). L. Van Look et al., ibid. 60, 6998 (1999). B. Y. Zhu et al, ibid. 64, 012504 (2001)

[2] R. S. Newrock et al., Solid State Phys. 54, 263 (2000).

[3] A. Schmid and W. Hauger, J. Low Temp. Phys. 11, 667 (1973)

[4] R. Thorel et al., J. Phys. (Paris) 34, 447 (1973); S. Bhattacharya and M.J. Higgins, Phys. Rev. Lett. 70, 2617 (1993); U. Yaron et al., Phys. Rev. Lett.73, 2748(1994); Nature (London) 376,753 (1995); A. Duarte et al., Phys. Rev. B53, 11336 (1996). F. Pardo et al., Phys. Rev. Lett.78, 4633 (1997); M. Marchevsky et al., ibid78, 531 (1997).

[5] C. Reichhardt et al., Phys. Rev. B 54, 16108 (1996); ibid. 57, 7937 (1998); ibid. 58, 6534 (1998); Phys. Rev. Lett. 782648 (1997); ibid. 82414 (1999); H. Fangohr, S.J. Cox, and P.A.J. de Groot, Phys. Rev. B 63, 064501 (2001); ibid 64, 064505 (2001).
[6] A.E. Koshelev and V.M Vinokur, Phys. Rev. Lett. 73, 3580 (1994).

[7] T. Giamarchi and P. Le Doussal, Phys. Rev. Lett. 76, 3408 (1996); Physica C 282,363(1997); Phys. Rev. B 57, 11356 (1998)

[8] G. Carneiro, J. Low Temp. Phys. 117, 1323 (1999); Phys. Rev. B 61, R 14661 (2000).

[9] G. Carneiro, cond-mat/0205461

[10] V.I. Marconi and D. Dominguez, Phys. Rev. Lett. 82, 4922 (1999); Phys. Rev B 63, 174509 (2001).

[11] V.I. Marconi et al., Phys. Rev. B 62, 4096 (2000).

[12] C. Reichhardt and G.T. Zimányi, Phys. Rev. B 61, 14354 (2000).

[13] E.H. Brandt, Rep. Prog. Phys. 58, 1465 (1995).

[14] P. Minnhagen, Rev. Mod. Phys. 59, 1001 (1987)

[15] See eg. D.R. Nelson, Phase transitions and Critical Phenomena, Vol. 7, C. Domb and M.S. Green, eds. (Academic Press,1983), Chap. 1.

[16] See eg. H. Risken, The Fokker-Planck Equation. Methods of Solution and Applications. (Springer,1989), Chap. 6. 